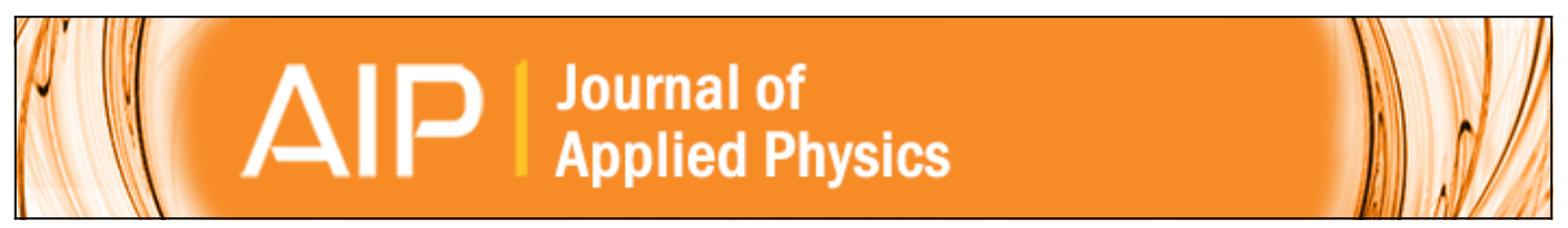

\title{
Thickness dependent charge transport in ferroelectric BaTiO3 heterojunctions
}

Pooja Singh, P. K. Rout, Manju Singh, R. K. Rakshit, and Anjana Dogra

Citation: Journal of Applied Physics 118, 114103 (2015); doi: 10.1063/1.4931158

View online: http://dx.doi.org/10.1063/1.4931158

View Table of Contents: http://scitation.aip.org/content/aip/journal/jap/118/11?ver=pdfcov

Published by the AIP Publishing

\section{Articles you may be interested in}

Strong oxygen pressure dependence of ferroelectricity in $\mathrm{BaTiO} / \mathrm{SrRuO} 3 / \mathrm{SrTiO} 3$ epitaxial heterostructures J. Appl. Phys. 114, 124101 (2013); 10.1063/1.4821643

Semiconductor layer thickness impact on optical and resistive switching behavior of pulsed laser deposited $\mathrm{BaTiO} / \mathrm{ZnO}$ heterostructures

Appl. Phys. Lett. 102, 212903 (2013); 10.1063/1.4809531

Temperature-dependent leakage current behavior of epitaxial Bi0.5Na0.5TiO3-based thin films made by pulsed laser deposition

J. Appl. Phys. 110, 103710 (2011); 10.1063/1.3660428

Electrical conduction transition and largely reduced leakage current in aluminum-doped barium strontium titanate thin films heteroepitaxially grown on $\mathrm{Ir} / \mathrm{MgO} / \mathrm{Si}$ ( 100 )

Appl. Phys. Lett. 86, 132902 (2005); 10.1063/1.1896448

Ferroelectric field effect in epitaxial LaVO 3 /( Ba,Sr )/ TiO $3 /(\mathrm{Pb}, \mathrm{La})(\mathrm{Zr}, \mathrm{Ti}) \mathrm{O} 3 /(\mathrm{La}, \mathrm{Sr}) \mathrm{CoO} 3$ heterostructures

J. Appl. Phys. 93, 4761 (2003); 10.1063/1.1560876

\section{AlP $\mid$ APL Photonics}

APL Photonics is pleased to announce Benjamin Eggleton as its Editor-in-Chief

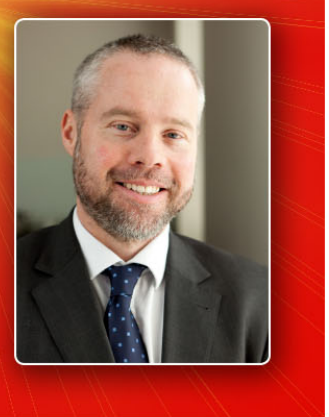




\title{
Thickness dependent charge transport in ferroelectric $\mathrm{BaTiO}_{3}$ heterojunctions
}

\author{
Pooja Singh, ${ }^{1,2, a)}$ P. K. Rout, ${ }^{2}$ Manju Singh, ${ }^{2}$ R. K. Rakshit,,${ }^{1,2}$ and Anjana Dogra ${ }^{1,2, a)}$ \\ ${ }^{1}$ Academy of Scientific and Innovative Research (AcSIR), CSIR-NPL Campus, Dr. K. S. Krishnan Marg, \\ New Delhi 110012, India \\ ${ }^{2}$ National Physical Laboratory, Council of Scientific and Industrial Research, Dr. K. S. Krishnan Marg, \\ New Delhi 110012, India
}

(Received 3 August 2015; accepted 6 September 2015; published online 21 September 2015)

\begin{abstract}
We have investigated the effect of ferroelectric barium titanate $\left(\mathrm{BaTiO}_{3}\right)$ film thickness on the charge transport mechanism in pulsed laser deposited epitaxial metal-ferroelectric semiconductor junctions. The current $(I)$-voltage $(V)$ measurements across the junctions comprising of 20-500 nm thick $\mathrm{BaTiO}_{3}$ and conducting bottom electrode $\left(\mathrm{Nb}: \mathrm{SrTiO}_{3}\right.$ substrate or $\mathrm{La}_{2 / 3} \mathrm{Ca}_{1 / 3} \mathrm{MnO}_{3}$ buffer layer) demonstrate the space charge limited conduction. Further analysis indicates a reduction in the ratio of free to trapped carriers with increasing thickness in spite of decreasing trap density. Such behaviour arises the deepening of the shallow trap levels $(<0.65 \mathrm{eV})$ below conduction band with increasing thickness. Moreover, the observed hysteresis in $I-V$ curves implies a bipolar resistive switching behaviour, which can be explained in terms of charge trapping and de-trapping process. C 2015 AIP Publishing LLC. [http://dx.doi.org/10.1063/1.4931158]
\end{abstract}

\section{INTRODUCTION}

Perovskite ferroelectrics (FEs) have been the subject of intensive research in recent years due to their potential application in various electronic devices like multilayer capacitor, ferroelectric tunnel junction, non-volatile memories, and electro-optic devices. ${ }^{1-7}$ These nanoscale devices require ferroelectric materials in thin film form with specific properties such as high Curie temperature, large spontaneous polarization, high dielectric constant, low dielectric loss, and large electro-optic coefficients. Amongst various ferroelectric oxides, $\mathrm{BaTiO}_{3}$ (BTO) is an excellent prototype with highest ferroelectric Curie temperature of $403 \mathrm{~K}$ and large value of spontaneous polarization for such applications. In recent years, tunnel electro-resistance as high as $10000 \%$ as well as resistance switching by five orders of magnitude in resistive switching device and memristor have been achieved using BTO as ferroelectric layer. ${ }^{5,8}$ Apart from ferroelectricity, the control of leakage current plays a crucial role in designing various electronic devices. While the leakage has detrimental effect on various electronic devices due to fatigue, imprint, resistance degradation, and breakdown, the photovoltaic devices require large leakage currents. ${ }^{9}$ Thus, the conduction mechanism in these systems become utmost important to understand and control the required properties of the micro/ nanoscale devices. Although BTO is considered as an insulator in bulk form, it is treated as a semiconductor due to the presence of free carriers during the charge transport in a device. In such metal-semiconductor junctions, four main charge transport processes are involved. Out of these, two mechanisms, i.e., Schottky emission (SE) and FowlerNordheim (F-N) tunnelling, are interface controlled phenomena while the other two, i.e., space charge limited current (SCLC) and Poole-Frenkel (P-F) emission, are bulk

\footnotetext{
${ }^{a)}$ Electronic addresses: poojasingh@nplindia.org and anjanad@nplindia.org.
}

controlled. Although there are several reports on the study of current $(I)$-voltage $(V)$ characteristics of BTO films, ${ }^{10-15}$ any one of the four mechanisms mentioned above or a combination of those have been reported to be responsible for charge conduction. Moreover, the forward and reverse bias current do not always follow same mechanism. The observation of such large variety of charge conduction indicates that various deposition techniques like molecular beam epitaxy, pulsed laser deposition (PLD), sputtering, etc., and the difference in growth parameters like substrate (or buffer), oxygen pressure, temperature, etc., play important role in these films. Apart from these, one more important parameter from the point of view of charge conduction is the thickness of ferroelectric BTO layer, whose effect has not been extensively studied till now. Boni et al. have reported the thickness dependent study of BTO, where the conduction results from a combination of thermionic injection at the interfaces and thermally activated hopping. ${ }^{16}$ The hopping mechanisms can arise due to the presence of oxygen vacancies in their films grown under oxygen pressure of $3 \times 10^{-5} \mathrm{mbar}$. The integration of ferroelectric BTO in oxide based electronic devices requires high oxygen pressure for good quality defect free growth of various oxide layers. Moreover, the pulsed laser deposition is a versatile technique for such growth involving multi-elemental compounds like $\mathrm{La}_{1-\mathrm{x}} \mathrm{Sr}_{\mathrm{x}} \mathrm{MnO}_{3}, \mathrm{LaNiO}_{3}, \mathrm{SrTiO}_{3}$ (STO), $\mathrm{CaTiO}_{3}$, etc. ${ }^{4,6,10,17,18}$ Therefore, the study of charge conduction in pulsed laser deposited BTO films under high oxygen pressure has been undertaken in this study. We have explored the effect of BTO thickness $(20-500 \mathrm{~nm})$ on charge conduction in the junction between ferroelectric BTO and the conducting electrodes. While the top electrode is kept as $\mathrm{Au}$, we have chosen two different bottom electrodes; namely, $n$-type $\mathrm{Nb}$ :STO and $p$-type $\mathrm{La}_{2 / 3} \mathrm{Ca}_{1 / 3} \mathrm{MnO}_{3}$ (LCMO), which assists the epitaxial growth of BTO layer. 


\section{EXPERIMENTAL DETAILS}

The epitaxial thin film of BTO has been deposited on (001) oriented $\mathrm{Nb}: \mathrm{STO}\left(1.4 \mathrm{wt}\right.$ \% $\mathrm{Nb}$ in $\mathrm{SrTiO}_{3}$ ) single crystal substrates using $\mathrm{KrF}$ excimer (wavelength $=248 \mathrm{~nm}$ ) based pulsed laser deposition technique. The thicknesses $(d)$ of BTO layer are 100, 200, 300, 400, and $500 \mathrm{~nm}$. Also, we have prepared 20, 50, and $100 \mathrm{~nm}$ thick BTO films on (001) STO substrate with $50 \mathrm{~nm}$ thick LCMO buffer layer. The films were deposited at a temperature of $750{ }^{\circ} \mathrm{C}$ under 0.5 mbar oxygen pressure and subsequently cooled in $1 \mathrm{~atm}$. oxygen pressure. The structural characterization was performed by X-Ray diffraction (XRD) with $\mathrm{Cu} \mathrm{K} \alpha$ source in $\theta-2 \theta, \omega$, and $\varphi$ modes. The surface morphology was investigated using atomic force microscopy (AFM). We have thermally evaporated $\mathrm{Au}(100 \mathrm{~nm})$ dots with $200 \mu \mathrm{m}$ diameter on the films, which serves as top electrodes. The bottom contact was taken from the back side of Nb:STO in case of BTO/ $\mathrm{Nb}$ :STO films. In case of buffered films, the top BTO layer was milled by Ar-ion milling, and the bottom contact was taken out from the exposed LCMO layer. The electrical measurements were performed in Lakeshore cryogenic probe station and closed cycle refrigerator, where the voltage bias was applied on the top electrode and the bottom electrode was kept as ground. All the measurements were performed at $300 \mathrm{~K}$.

\section{RESULTS AND DISCUSSION}

\section{A. Structural characterization}

Figure 1(a) displays the $\theta-2 \theta \mathrm{X}$-ray diffraction patterns for $100 \mathrm{~nm}$ thick BTO grown on $\mathrm{Nb}$ :STO substrate and LCMO $(50 \mathrm{~nm})$ buffered STO substrate. We observe the presence of $(00 l)$ oriented BTO peaks, which indicate the $c$-axis oriented epitaxial growth of BTO layer in both kinds of films. The high crystalline quality of these films is revealed from $\omega$-scans with full width at half maximum (FWHM) less than $0.55^{\circ}-0.68^{\circ}$ [see Figs. 1(b) and 1(c)]. The

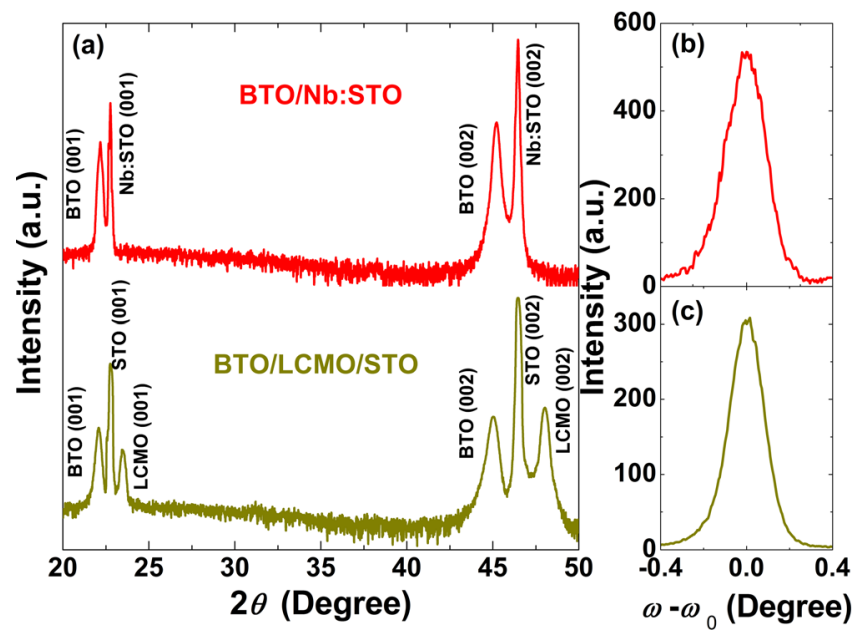

FIG. 1. (a) The $\theta-2 \theta$ XRD pattern for BTO $(100 \mathrm{~nm}) / \mathrm{Nb}: \mathrm{STO}$ and BTO $(100 \mathrm{~nm}) / \mathrm{LCMO}(50 \mathrm{~nm}) / \mathrm{STO}$ heterostructures. The rocking curves about (001) BTO peak for (b) BTO/Nb:STO with $\omega_{0}=22.61^{\circ}$ and (c) BTO/ LCMO/STO with $\omega_{0}=22.55^{\circ}$. average crystallite size $(D)$ can be calculated from Scherrer formula: $D=0.94 \lambda / \beta \cos \theta$, where $\lambda$ is $\mathrm{X}$-ray wavelength, $\theta$ is Bragg angle, and $\beta$ is FWHM in radian. The calculated crystallite sizes of the BTO layer are $8-15 \mathrm{~nm}$. The epitaxial growth of the BTO layer further confirmed by $\varphi$-scans (not shown here) about (111) peak represents four symmetric peaks observed at every $90^{\circ}$ rotation due to four-fold symmetry of the layers. Moreover, the peaks of each component of the film (i.e., BTO, LCMO, and STO) are present at exact same $\varphi$ value, which implies an epitaxial relation of the form: [100] BTO || [100] LCMO || [100] STO. Thus, our crystalline films will have lesser defects and disorder (thereby lesser leakage currents) as compared to polycrystalline films. Now, we comment on the role of thickness on the structural modification of BTO layer due to strain effect. The lattice mismatches $\left[\left(a_{B T O}-a_{\text {sub }}\right) / a_{\text {sub }}\right]$ of BTO $(a=3.994 \AA$, $c=4.038 \AA)$ with $\mathrm{Nb}: \mathrm{STO} \quad(a=3.905 \AA)$ and LCMO $(a=3.86 \AA)$ are $+2.3 \%$ and $+3.3 \%$, respectively. Thus, the BTO layer is under in-plane biaxial compressive strain, which enhances out-of-plane lattice constant (c). But, in our heterostructures, the thick BTO layer is expected to be fully relaxed. We have verified this by extracting the $c$ from $\theta-2 \theta$ XRD scans, which reveals expected thickness independent nature of $c$. Moreover, the fabrication of good quality junctions requires smooth topography and low roughness of the layers. While the AFM images of the films confirm a uniform and homogeneous surface with maximum root-meansquare roughness of $2-3 \mathrm{~nm}$, similar roughness values are obtained from $\mathrm{X}$-ray reflectivity measurements.

\section{B. Current ( () -voltage ( $V$ ) characteristics}

The room temperature current density $(J)$-voltage $(V)$ characteristics of all $\mathrm{Au} / \mathrm{BTO} / \mathrm{Nb}: \mathrm{STO}$ and $\mathrm{Au} / \mathrm{BTO} / \mathrm{LCMO}$ heterostructures are presented in Fig. 2. Clearly, all the curves demonstrate rectifying behavior with the reduction in the current densities with increasing film thickness. There are two noticeable differences in $J-V$ data of these heterostructures. First, the leakage current density in BTO/LCMO

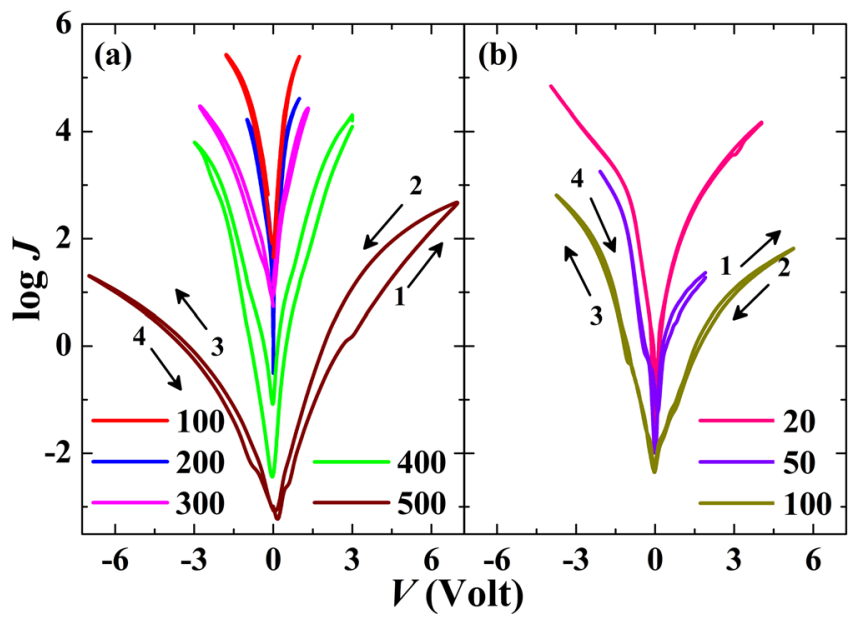

FIG. 2. The semi-logarithmic $J-V$ curves for (a) Au/BTO/Nb:STO with $d=100,200,300,400$, and $500 \mathrm{~nm}$ as well as (b) Au/BTO/LCMO with $d=20,50$, and $100 \mathrm{~nm}$. The arrows and numbers indicate the voltage sweep direction and sequence. 
is lower than that in $\mathrm{BTO} / \mathrm{Nb}: \mathrm{STO}$ for same thickness. Second, the $J$ for positive voltage bias is higher than that for negative polarity in $\mathrm{BTO} / \mathrm{Nb}: \mathrm{STO}$; while opposite trend is observed for BTO/LCMO. Since the top electrode is same in both types of structures, the differences arise only due to different bottom electrodes. The first difference arises due to the difference between the work function of metal (bottom electrode) and electron affinity of the semiconductor (BTO). This results in the formation of barrier at the interface and the depletion region (caused due to the flow of carriers to equalize the Fermi level) near the interface. Assuming $n$ - type semiconducting nature of BTO, the metal with higher work function will form higher barrier. In our films, the work function of LCMO $(4.8 \mathrm{eV})$ is higher than that of $\mathrm{Nb}$ :STO $(4.1 \mathrm{eV})$, and, thus, lower leakage is observed for BTO/LCMO due to higher barrier at the interface. The second difference stems from the type of charge carriers present in the bottom electrodes. While $\mathrm{Nb}$ :STO is $n$-type with more electrons (majority carriers) compared to BTO, LCMO is $p$-type with holes as majority carriers. Thus, in BTO/ $\mathrm{Nb}$ :STO, the positive polarity makes bottom electrode $\mathrm{Nb}$ :STO to inject electrons into BTO, which results in higher current compared to the case of negative polarity. In contrast, the negative polarity in $\mathrm{BTO} / \mathrm{LCMO}$ drives the electron into LCMO causing higher current. Another important feature of these $J-V$ data is the presence of resistive switching, which results in two different resistance states (high resistive state "HRS" and low resistive state "LRS") due to hysteresis during voltage sweeps. We will discuss about this feature in more detail in the later part of this manuscript.

The conduction in these metal-ferroelectric heterostructures is determined by the mechanisms related to both bulk of the film and the interface as discussed before. Also, there is a possibility that one of them can dominate the charge transport and thereby suppressing the other effects. To determine the dominant conduction mechanism in these junctions, we begin more rigorous analysis of our data using various models. First, we can rule out the F-N mechanism as the electron tunneling across such thick $(>20 \mathrm{~nm})$ BTO layer is unlikely. The second interface controlled process is the Schottky emission. The current density in metalsemiconductor Schottky model can be expressed as ${ }^{19}$

$$
J=A T^{2} \exp \left[\frac{-q}{k T}\left(\Phi_{B}-\sqrt{\frac{q E}{4 \pi \varepsilon_{0} \varepsilon_{d y}}}\right)\right]
$$

where $q$ is the electronic charge, $k$ is Boltzmann constant, $T=300 \mathrm{~K}, \Phi_{B}$ is the Schottky barrier height, $\varepsilon_{0}=8.854$ $\times 10^{-12} \mathrm{C}^{2} \mathrm{~N}^{-1} \mathrm{~m}^{-2}$ is vacuum permittivity, and $\varepsilon_{d y}$ is dynamic dielectric constant. The Richardson constant $(A)$ is defined as: $A=\left(4 \pi k q m^{*} / h^{3}\right)=120\left(m^{*} / m\right)$, where $m$ is the electronic mass and $m^{*}=5 m$ is the effective mass of electron. ${ }^{20}$ Figure 3(a) displays $\ln J$ vs. ${ } E$ plot for Au/BTO/Nb:STO and $\mathrm{Au} / \mathrm{BTO} / \mathrm{LCMO}$ junctions. Clearly, the fits according to Eq. (1) cannot account for the whole voltage range, which implies that this may not be the conduction mechanism in these junctions. Still we went ahead with the fitting to extract two important parameter; dynamic dielectric constant and

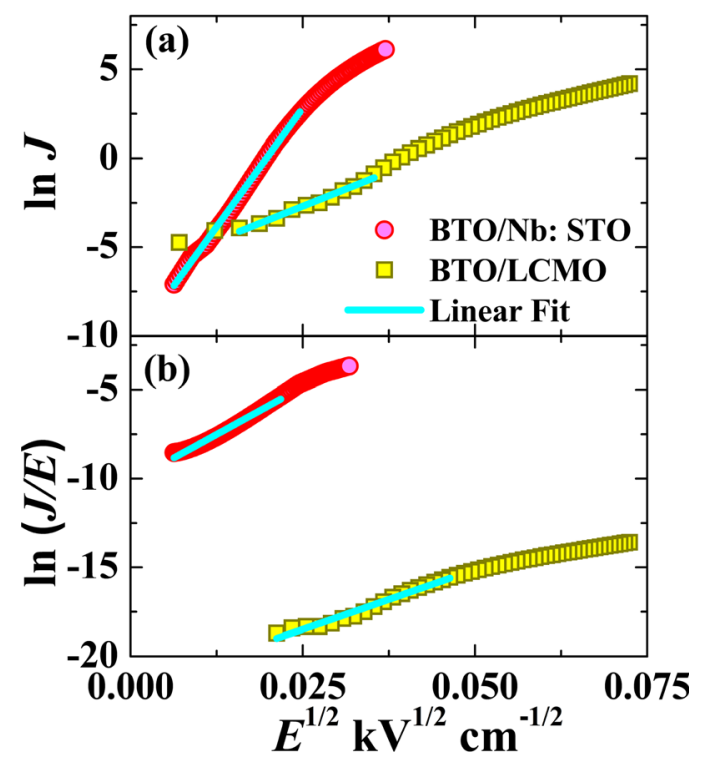

FIG. 3. The positive polarity high resistive state data of BTO $(100 \mathrm{~nm}) / \mathrm{Nb}$ : STO and BTO $(100 \mathrm{~nm}) / \mathrm{LCMO}$ along with the linear fits according to (a) Schottky emission and (b) Poole-Frenkel emission.

barrier height. The extracted values of dynamic dielectric constant are around $0.08-0.16$, which is quite low compared to previously reported value of $6.3 .^{12}$ Our estimated barrier height for $\mathrm{BTO} / \mathrm{Nb}: \mathrm{STO}$ structures increases from 0.45 to $0.7 \mathrm{eV}$ with increasing BTO layer thickness and the $\Phi_{B}$ for $\mathrm{BTO} / \mathrm{LCMO}$ increases from 0.5 to $0.65 \mathrm{eV}$. Moreover, the variation of $\Phi_{B}$ with thickness is contradictory to the interfacial nature of SE mechanism. Here, we mention a thickness dependent study of $\Phi_{B}$ in $\mathrm{Pb}(\mathrm{Zr}, \mathrm{Ti}) \mathrm{O}_{3}$ by Lin et al. ${ }^{21}$ They have proposed that the reduction in the film thickness results in enhanced band bending and thereby increasing the barrier height, which is opposite to our experimental results. Although we cannot deny the fact that there will be some contribution from SE in $I-V$ data for low enough electric fields, the above observations negate the possibility of interface dominated conduction in our junctions. Coming to bulk dominated mechanism, we first tried to explain the $J-V$ data using P-F mechanism, which is governed by the following expression: ${ }^{19}$

$$
J=q \mu N_{c} E \exp \left[\frac{-q}{k T}\left(\Phi_{T}-\sqrt{\frac{q E}{\pi \varepsilon_{0} \varepsilon_{d y}}}\right)\right],
$$

where $\mu$ is the electron mobility, $N_{c}$ is effective density of state in conduction band, and $\Phi_{T}$ is the barrier height of trap. The fits [see Fig. 3(b)] to the data in the limited range of voltage yield a wide variation in the values of $\varepsilon_{d y}$ ranging from 0.5 to 8 . Also, we observe an increasing trend for $\Phi_{T}$ with increasing thickness $(0.33-0.62 \mathrm{eV}$ for $\mathrm{BTO} / \mathrm{Nb}$ :STO and $0.55-0.67 \mathrm{eV}$ for BTO/LCMO). Therefore, we can safely rule out the P-F mechanism in these films. Now, we consider the last possible conduction mechanism viz., SCLC, where the injected carrier density from the metal electrodes exceeds the free carrier density of the bulk BTO. In this scenario, the SCLC current density in the presence of traps is given as ${ }^{19}$ 


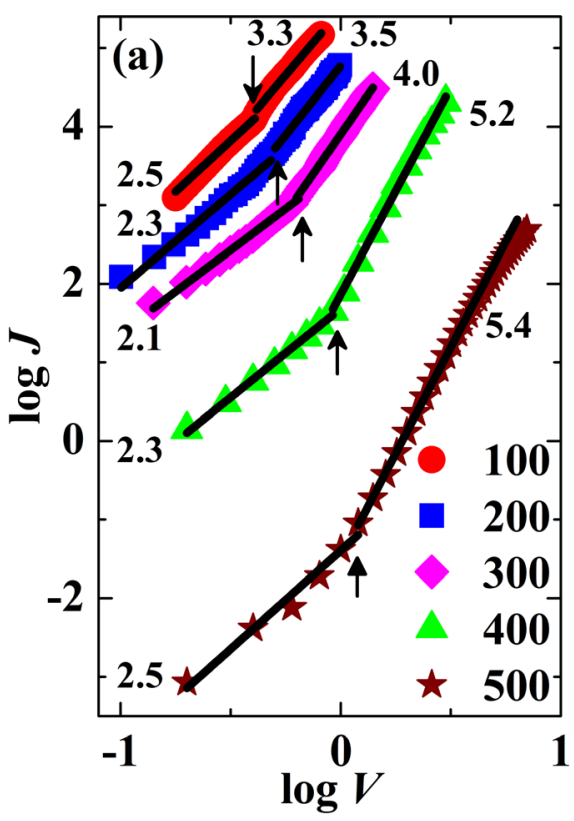

$$
J=\frac{9 \theta \mu \varepsilon_{r} \varepsilon_{0} V^{2}}{8 d^{3}},
$$

where $\theta$ is the ratio of free to trapped carriers and $\varepsilon_{r}$ is the static dielectric constant. Here, we have used previously reported value of $\varepsilon_{r}=185$ and $\mu=10^{-5} \mathrm{~m}^{2} \mathrm{~V}^{-1} \mathrm{~s}^{-1} \cdot{ }^{10,20}$ This expression qualitatively explains the reduction of current density with increasing as shown in Fig. 2. With the increase in the voltage, the injected carriers from the electrode start filling the traps present in the bulk of the film. At a certain voltage $V_{T F L}$ (trap filled limit), all the traps become filled and the current density rises rapidly. The $V_{T F L}$ divides the $J-V$ data into two different regions, as shown in Fig. 4. The lower electric field data show a quadratic relationship between $J$ and $V$ [Eq. (3)], which is a hallmark of SCLC. On the contrary, the higher field regions have much higher slope, which is due to continuous distribution of the traps. ${ }^{22}$ Moreover, the trap density $\left(N_{t}\right)$ can determined from $V_{T F L}$ using the relation ${ }^{19}$

$$
N_{t}=\frac{9 \varepsilon_{r} \varepsilon_{0} V_{T F L}}{8 q d^{2}} .
$$

In a heterostructure, the regions near the interfaces will have larger number of traps as compared to the bulk part of the film. Vianello et al. have reported the spatial distribution of stress generated traps, which decrease from the interface toward the bulk of the dielectric. ${ }^{23}$ Therefore, the trap density is expected to be more for thinner films as observed from our extracted values of $N_{t}$ [see Fig. 5(a)]. We have also extracted the $\theta$ from the intercept of linear fits to $\log J-\log V$ data. The thickness dependence of $\theta$ is presented in Fig. 5(b). Clearly, the $\theta$ decreases with increasing thickness in spite of decreasing $N_{t}$, which seems contradictory at first glance. Moreover, the values of $\theta$ for BTO/LCMO are order of magnitude smaller than those for BTO/Nb:STO. To understand these, we have to consider the depth of the trap level in addition to $N_{t}$. The trapping of the carriers is a thermal process and, thus, it depends on the energy difference between the
FIG. 4. The $\log J-\log V$ plot for (a) $\mathrm{BTO} / \mathrm{Nb}: \mathrm{STO}$ and (b) BTO/LCMO. The values of slopes to the linear fit are written in the figure. The arrows indicate the voltage $\left(V_{T F L}\right)$ at the trap filled limit. Here, we have shown the positive polarity high resistive state data. The other branches of $J-V$ curve show similar behavior.

政) and the conduction band $\left(E_{c}\right)$, which is called activation energy $E_{A}=E_{c}-E_{t}$. The $\theta$ is related to $E_{A}$ by the expression: $\theta=\left(N_{c} / g N_{t}\right) \exp \left[-\left(E_{c}-E_{t}\right) / k T\right]$, where $g=2$ is degeneracy factor and $N_{c}$ is effective density of state in conduction band. ${ }^{19}$ The $N_{c}$ can be taken as $10^{21} \mathrm{~cm}^{-3}$ as observed in these systems. ${ }^{24}$ The activation energies extracted from the expression are less than $0.65 \mathrm{eV}$ in these heterostructures as compared to band gap (3.2 eV) of BTO as shown in inset of Fig. 6. Such low activation energy implies that the trap energy level lies close enough to the conduction band and, thus, these traps are called "shallow traps." In such scenario, the electrons in trap level and conduction band are in thermal equilibrium and the charge conduction follows thermally activation process as assumed before. Moreover, we observe an enhancement in $E_{A}$ (or the deepening of the trap level) with increasing thickness [Fig. 6]. Therefore, more number of the trapped carriers is freed due to lower $E_{A}$ and higher $N_{t}$, which will result in higher $\theta$ for thinner films. Moreover, the values of $E_{A}$ for $\mathrm{BTO} / \mathrm{LCMO}$ are larger in comparison to those for BTO/ $\mathrm{Nb}: \mathrm{STO}$, which results in lower $\theta$ in case of BTO/LCMO.

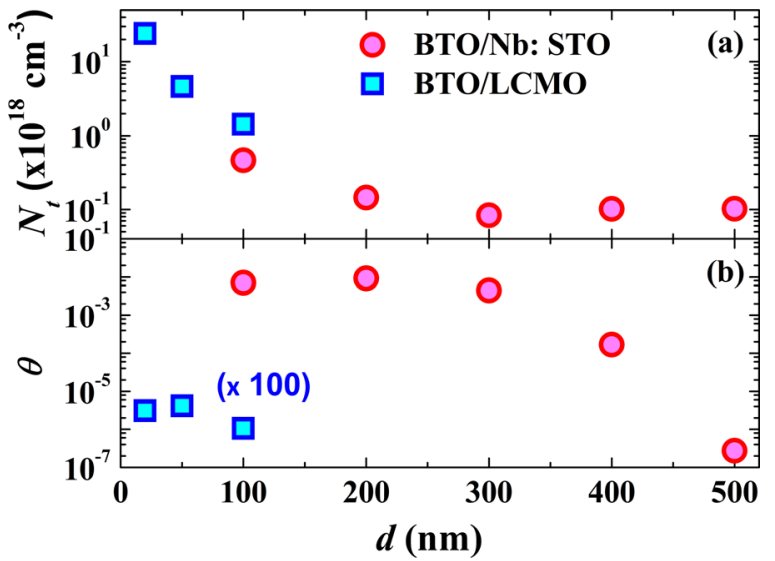

FIG. 5. The thickness dependence of (a) $N_{t}$ and (b) $\theta$ for BTO/Nb:STO and BTO/LCMO. The values of $\theta$ for BTO/LCMO are multiplied by a factor of 100 


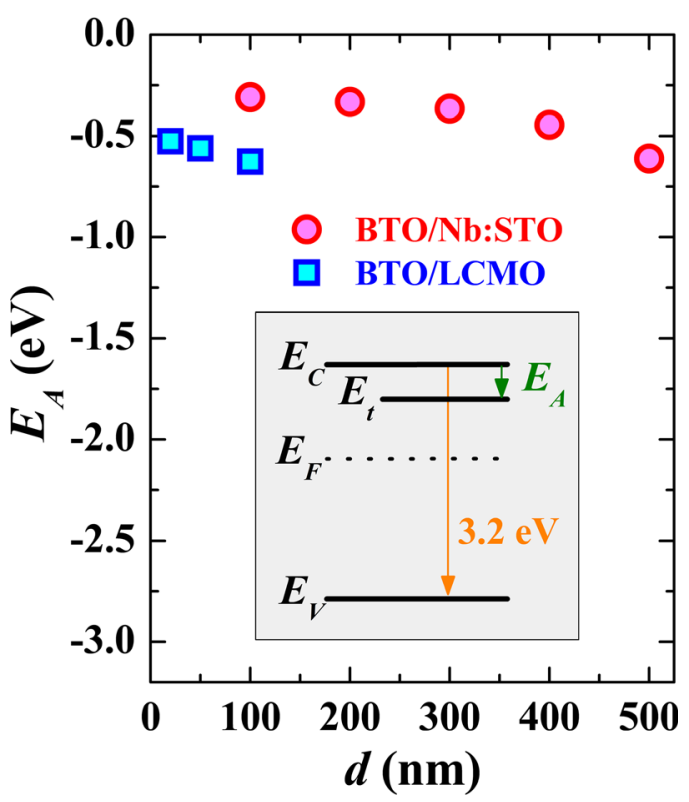

FIG. 6. The activation energy $\left(E_{A}\right)$ for BTO/Nb:STO and BTO/LCMO heterostructures as a function of BTO layer thickness. The inset shows the schematic band diagram showing conduction band $\left(E_{C}\right)$, trap energy level $\left(E_{t}\right)$, Fermi level $\left(E_{F}\right)$, and valence band $\left(E_{V}\right)$.

Here, we also point out that the conduction in our films is dominated by SCLC. This in contrast to the thermally activated hopping dominated conduction for the BTO films grown in lower oxygen pressures $\left(3 \times 10^{-5} \mathrm{mbar}\right)$.

Finally, we discuss about the bipolar RS seen in the $J-V$ data. Though the hysteresis loop is more pronounced for thicker films, the change in resistance is not abrupt implying feeble switching behavior. Such weak RS has been reported in PLD grown oxygen deficient $\mathrm{SrRuO}_{3} / \mathrm{BTO} / \mathrm{SrRuO}_{3}$ heterostructure, where oxygen vacancy migration is responsible for observed $\mathrm{RS} .{ }^{25} \mathrm{Li}$ et al. have investigated the role of oxygen deposition pressure on RS in BTO. ${ }^{26}$ With increasing pressure, the nature of RS changes from bipolar RS with LRS to HRS switching during positive cycle (due to oxygen vacancy migration) to bipolar RS with HRS to LRS switching during positive cycle (due to polarization reversal) and then to unipolar weak RS (due to dielectric relaxation current). Even unipolar RS has been observed in doped BTO films due to conductive filament creation/rupture. ${ }^{27}$ Moreover, the forming processes or the creation of an additional oxide layer at the interface are required to observed RS in many BTO heterostructures. ${ }^{13,14,28}$ Above discussion provides number of possible origin of RS in BTO films. We can rule out the possibility of oxygen vacancy and conductive filament formation due to the following reasons; (1) our films are undoped, (2) they are grown in high oxygen pressure, and (3) we have not performed any forming process before starting the measurement. In case of polarization reversal mediated RS, the barrier height decreases at one interface towards the direction of polarization while it increases at the other interface. Since SCLC is the dominant process in our films, we do not observe any such barrier modulation. The extracted Schottky barrier heights for BTO $(500 \mathrm{~nm}) /$ $\mathrm{Nb}: \mathrm{STO}$ are $0.64 \mathrm{eV}$ for positive polarity and $0.61 \mathrm{eV}$ for negative polarity. Moreover, the barrier height increases with increasing thickness for both the polarities. This is also contradictory to the polarization reversal scenario, where one barrier height should increase with increasing thickness while the other should decrease. Since we do not see any change in $I-V$ data with voltage sweep rate, our results deal with steady state conduction with no dielectric relaxation current. We believe that the observed RS in our films is due to the trapping and de-trapping of the charge carriers as observed in other ferroelectric systems like $\mathrm{LaFeO}_{3}-\mathrm{PbTiO}_{3}$ and $(\mathrm{Pb}, \mathrm{La})(\mathrm{Zr}, \mathrm{Ti}) \mathrm{O}_{3} \cdot{ }^{15,29}$ When positive bias is applied in $\mathrm{BTO} / \mathrm{Nb}$ :STO junctions, the electrons are injected from $\mathrm{Nb}$ :STO (bottom electrode) into BTO and starts filling the traps. This results in slow increase of current with voltage, which forms the HRS for $0 \rightarrow+\mathrm{V}$. While coming back from a positive voltage $(+\mathrm{V} \rightarrow 0)$, the filled traps do not hinder the conduction and thus LRS is attained. Further reduction in bias $(0 \rightarrow-\mathrm{V})$ starts de-trapping of the carriers and switches it back into HRS state when $-\mathrm{V} \rightarrow 0$. Such anti-clockwise direction of hysteresis loop is known as negative bipolar RS. In contrast, the BTO/LCMO junctions show positive bipolar RS since the holes are the majority carriers in LCMO. Another feature of observed RS is the reduction of RS with decreasing thickness. This is related to the depth of trap level, which reduces with decreasing thickness [Fig. 6]. Thus, the trapping (or de-trapping) of the carriers happens with much lower biases and thereby reducing the hysteresis.

\section{CONCLUSION}

The effect of thickness on the conduction mechanism in epitaxial BTO films grown on conducting Nb:STO substrate and LCMO buffered STO substrate has been studied extensively. Space charge limited conduction dominates in these metals-BTO junctions. While the trap density decreases with increasing thickness, the ratio of free to trapped carriers also reduces, this is an effect of increasing activation energy of thermally mediated trapping process. Still these shallow traps lie near the conduction band edge within energy range of $0.65 \mathrm{eV}$. Furthermore, the conduction across BTO/ $\mathrm{Nb}$ :STO junction is characterised by higher $\theta$ and lower $E_{A}$ in comparison to $\mathrm{BTO} / \mathrm{LCMO}$ junction. We have also observed negative bipolar resistive switching in BTO/ $\mathrm{Nb}$ :STO while positive switching is observed for BTO/ LCMO. The observed switching behaviour and its thickness dependence were explained in terms of the trapping and detrapping of the carriers.

\section{ACKNOWLEDGMENTS}

The authors would like to thank R. C. Budhani for his valuable suggestions. P.S. and P.K.R. acknowledge DST for financial support.

${ }^{1}$ D. J. Kim, H. Lu, S. Ryu, C.-W. Bark, C. B. Eom, E. Y. Tsymbal, and A. Gruverman, Nano Lett. 12, 5697 (2012).

${ }^{2}$ R. Soni, A. Petraru, P. Meuffels, O. Vavra, M. Ziegler, S. K. Kim, D. S. Jeong, N. A. Pertsev, and H. Kohlstedt, Nat. Commun. 5, 5414 (2014).

${ }^{3}$ D. J. Kim, J. Y. Jo, Y. S. Kim, Y. J. Chang, J. S. Lee, J. G. Yoon, T. K. Song, and T. W. Noh, Phys. Rev. Lett. 95, 237602 (2005). 
${ }^{4}$ C. Li, L. Huang, T. Li, W. Lü, X. Qiu, Z. Huang, Z. Liu, S. Zeng, R. Guo, Y. Zhao, K. Zeng, M. Coey, J. Chen, Ariando, and T. Venkatesan, Nano Lett. 15, 2568 (2015)

${ }^{5}$ Y. W. Yin, J. D. Burton, Y. M. Kim, A. Y. Borisevich, S. J. Pennycook, S. M. Yang, T. W. Noh, A. Gruverman, X. G. Li, E. Y. Tsymbal, and Q. Li, Nat. Mater. 12, 397 (2013).

${ }^{6}$ A. Chanthbouala, V. Garcia, R. O. Cherifi, K. Bouzehouane, S. Fusil, X. Moya, S. Xavier, H. Yamada, C. Deranlot, N. D. Mathur, M. Bibes, A. Barthélémy, and J. Grollier, Nat. Mater. 11, 860 (2012).

${ }^{7}$ K. J. Choi, M. Biegalski, Y. L. Li, A. Sharan, J. Schubert, R. Uecker, P. Reiche, Y. B. Chen, X. Q. Pan, V. Gopalan, L.-Q. Chen, D. G. Schlom, and C. B. Eom, Science 306, 1005 (2004).

${ }^{8}$ Z. Wen, C. Li, D. Wu, A. Li, and N. Ming, Nat. Mater. 12, 617 (2013).

${ }^{9}$ C. J. Won, Y. A. Park, K. D. Lee, H. Y. Ryu, and N. Hur, J. Appl. Phys. 109, 084108 (2011)

${ }^{10}$ W. Zhang, Y. Gao, L. Kang, M. Yuan, Q. Yang, H. Cheng, W. Pan, and J. Ouyang, Acta Mater. 85, 207 (2015).

${ }^{11}$ T. J. Zhang, R. K. Pan, Z. J. Ma, M. G. Duan, D. F. Wang, and M. He, Appl. Phys. Lett. 99, 182106 (2011).

${ }^{12}$ R. K. Pan, T. J. Zhang, J. Z. Wang, Z. J. Ma, J. Y. Wang, and D. F. Wang, J. Alloys Compd. 519, 140 (2012).

${ }^{13}$ R. K. Pan, T. J. Zhang, J. Y. Wang, J. Z. Wang, D. F. Wang, and M. G. Duan, Thin Solid Films 520, 4016 (2012).

${ }^{14}$ C. H. Jung, S. I. Woo, Y. S. Kim, and K. S. No, Thin Solid Films 519, 3291 (2011).
${ }^{15}$ Y. Watanabe, D. Sawamura, and M. Okano, Solid State Ionics 108, 109 (1998).

${ }^{16}$ A. G. Boni, I. Pintilie, L. Pintilie, D. Preziosi, H. Deniz, and M. Alexe, J. Appl. Phys. 113, 224103 (2013).

${ }^{17}$ H. N. Lee, H. M. Christen, M. F. Chisholm, C. M. Rouleau, and D. H. Lowndes, Nature 433, 395 (2005).

${ }^{18}$ S. Kundu, M. Clavel, P. Biswas, B. Chen, H. C. Song, P. Kumar, N. N. Halder, M. K. Hudait, P. Banerji, M. Sanghadasa, and S. Priya, Sci. Rep. 5, 12415 (2015).

${ }^{19}$ F. C. Chiu, Adv. Mater. Sci. Eng. 2014, 578168.

${ }^{20}$ W. Heywang, J. Mater. Sci. 6, 1214 (1971).

${ }^{21}$ C. H. Lin, P. A. Friddle, C. H. Ma, A. Daga, and H. Chen, J. Appl. Phys. 90, 1509 (2001)

${ }^{22}$ A. Rose, Phys. Rev. 97, 1538 (1955).

${ }^{23}$ E. Vianello, F. Driussi, D. Esseni, L. Selmi, F. Widdershoven, and M. J. V. Duuren, IEEE Trans. Electron Devices 54, 1953 (2007).

${ }^{24}$ S. T. Chang and J. Y. Lee, Appl. Phys. Lett. 80, 655 (2002).

${ }^{25}$ Y. A. Park, K. D. Sung, C. J. Won, J. H. Jung, and N. Hur, J. Appl. Phys. 114, 094101 (2013).

${ }^{26}$ M. Li, J. Zhou, X. Jing, M. Zeng, S. Wu, J. Gao, Z. Zhang, X. Gao, X. Lu, J. M. Liu, and M. Alexe, Adv. Electron. Mater. 1, 1500069 (2015).

${ }^{27}$ Z. Yan, Y. Guo, G. Zhang, and J. M. Liu, Adv. Mater. 23, 1351 (2011).

${ }^{28}$ C. Wang and M. H. Kryder, J. Phys. D: Appl. Phys. 41, 245301 (2008).

${ }^{29}$ P. Zhang, C. Gao, F. Lv, Y. Wei, C. Dong, C. Jia, Q. Liu, and D. Xue, Appl. Phys. Lett. 105, 152904 (2014). 